\title{
Le fratture toraco-lombari: il ruolo della risonanza magnetica
}

A.Zerbi,G.Pozzi, P.Stradiotti

IRCCS Istituto Ortopedico Galeazzi, Milano

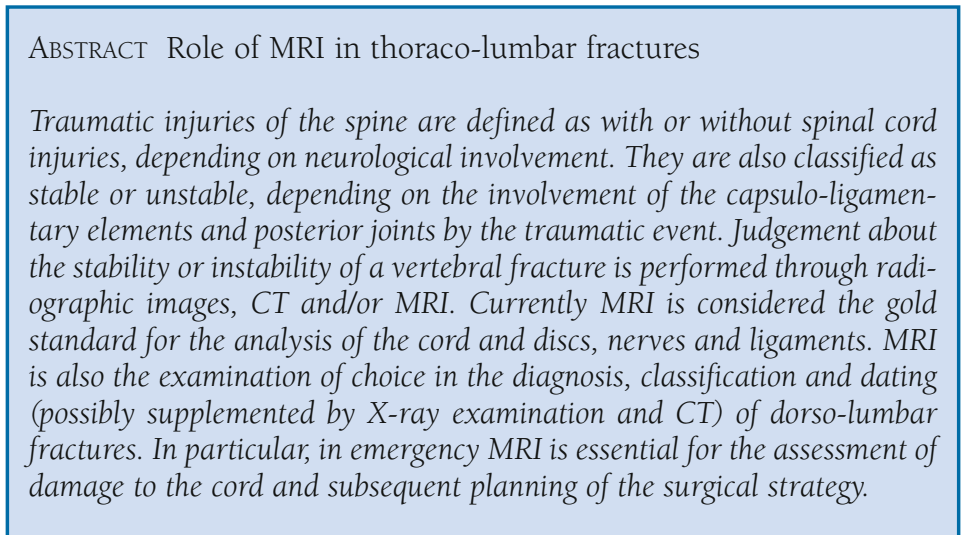

Statisticamente le lesioni traumatiche della colonna vertebrale si realizzano con maggiore frequenza (60\% dei casi) a livello della zona di passaggio tra colonna toracica e lombare (la cosiddetta giunzione toraco-lombare), essenzialmente per motivi legati alla meccanica vertebrale: - la giunzione toraco-lombare è una zona di passaggio fra un tratto rachideo piuttosto rigido (la colonna toracica, vincolata nei suoi movimenti intervertebrali dall'apparato costale) e uno (il tratto lombare) con spiccate caratteristiche di movimento

- è posta fra un tratto di colonna in cifosi (il rachide toracico) e uno in lordosi (il rachide lombare)

- è un punto di transizione fra un tratto di colonna in cui il movimento intervertebrale è costituito prevalentemente da un movimento di rotazione (il rachide toracico) e un tratto (il vimento fisiologicamente più rappresentato è quello di flesso-estensione.

In intimo rapporto con la colonna vertebrale vi è il midollo spinale, che può essere coinvolto seguente sintomatologia motoria e/o sensitiva.

Le lesioni traumatiche della colonna vertebrale con coinvolgimento neurologico vengono definite mieliche; le fratture in cui non vi sia tale coinvolgimento sono denominate amieliche. Esse vengono inoltre classificate in stabili e instabili, a seconda del coinvolgimento o meno degli elementi capsulo-legamentosi e articolari posteriori da parte dell'evento traumatico.

Una lesione traumatica della colonna vertebrale viene definita tura del corpo, si evidenziano le-

\section{$\nabla$ M.B. Gallazzi}

\section{Letture consigliate}

Baker LL, Goodman SB, Perkash I et al (1990) Benign versus pathologic compression fractures of vertebral bodies: assessment with conventional spin-echo, chemical shift, and STIR MR imaging. Radiology 174:495-502

Brant-Zawadzki M, Miller EM, Federle MP (1981) CT in evaluation of spine trauma. AJR Am J Roentgenol 136:369-375

Daffner RH, Deeb ZL, Goldberg AL et al (1990) The radiologic assessment of post-traumatic vertebral stability. Skeletal Radiol 19:103-108

Denis F (1983) The three column spine and its significance in the classification of acute thoracolumbar spinal injuries. Spine 8:817-831

Eastell R, Cedel SL, Wahner HW et al (1991) Classification of vertebral fractures. J Bone Mineral Res 6:207-215

Enzmann DR, DeLaPaz RL, Rubin JB (eds) (1990) Magnetic resonance of the spine. Mosby, St. Louis:376-366

El-Khoury GY, Whitten GC (1993) Trauma to the upper thoracic spine: anatomy, biomechanics, and unique imaging features. AJR Am J Roentgenol 160:95-102

El-Khoury GY, Kathol MH, Daniel WW (1995) Imaging of acute injuries of the cervical spine: value rachide lombare) in cui il modall'evento traumatico, con coninstabile quando, oltre alla frat-

DOl 10.1007/s10261-011-0017-3

sioni degli elementi molli o scheletrici dell'arco posteriore. In questo caso è elevata la probabilità di olistesi multidirezionale residua della vertebra, che andrà quindi controllata con tecniche chirurgiche. Nel caso invece di frattura stabile potrà essere sufficiente un trattamento non chirurgico.

Il giudizio circa la stabilità o l'instabilità di una frattura vertebrale viene dato tramite le immagini radiografiche, $\mathrm{TC}$ e/o di risonanza magnetica (RM), che individuano le varie strutture vertefondimenti diagnostici consen-

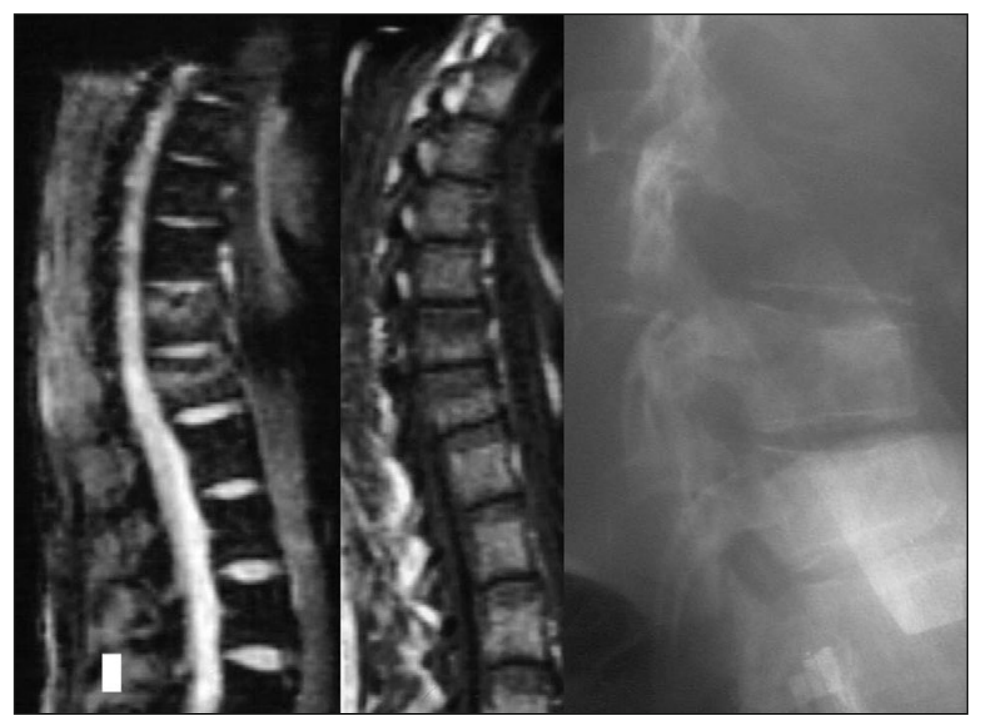

Fig. 1. Riconoscimento, classificazione e datazione delle fratture
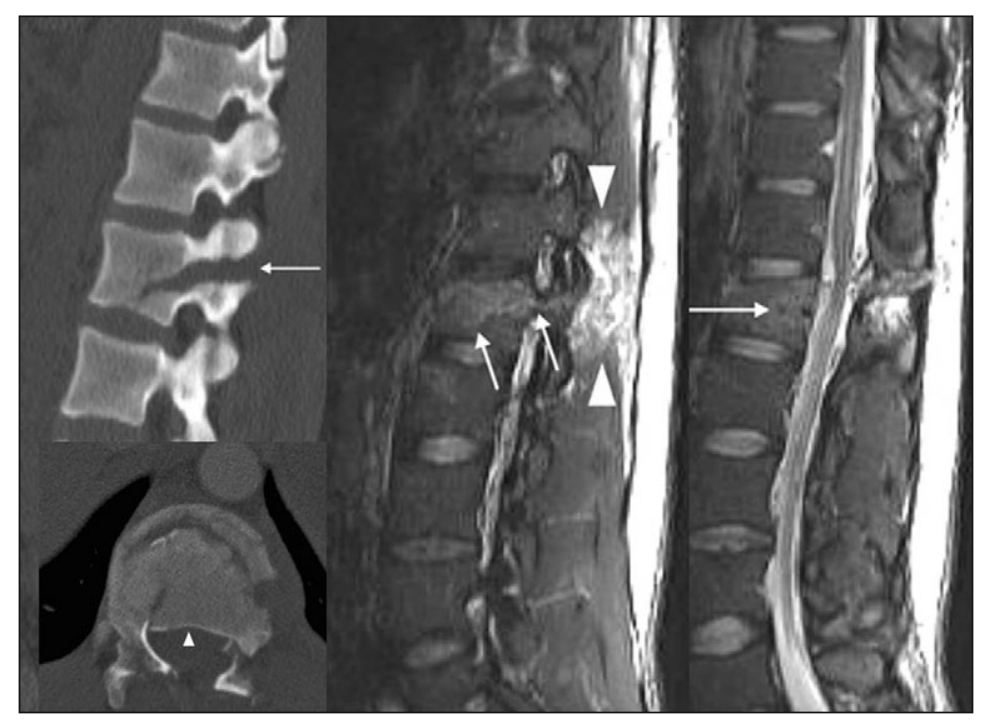

Fig. 2. Frattura instabile amielica ("Chance fracture")

racolumbar fractures. An analysis of one hundred consecutive cases and a new classification. J Bon Joint Surg Am 65:461-473

Melton LJ 3rd, Kan SH, Frye MA et al (1989) Epidemiology of vertebral fractures in women. Am J Epidemiol 129:1000-1011

Yuh WT, Zachar CK, Barloon TJ et al (1989) Vertebral compression fractures: distinction between benign and malignant causes with MR imaging. Radiology 172:215 218

Wiener SN, Neumann DR, Rzeszotarski MS (1989) Comparison of magnetic resonance imaging and radionuclide bone imaging of vertebral fractures. Clin Nucl Med 14:666-670

tono di classificare le differenti fratture, in ragione delle caratteristiche delle lesioni (ossee e legamentose) che sono causate dalle diverse meccaniche lesionali. Attualmente la RM è considerail gold standard" per l'analisi dei dischi, dei nervi e dei legamenti. È estremamente sensibile nel riconoscere le fratture, anche se non così precisa come la TC, ne permette la datazione e ne facilita la classificazione (Fig. 1). permette di identificare l'edema osseo con una sensibilità del $100 \%$ e consente inoltre di valu-

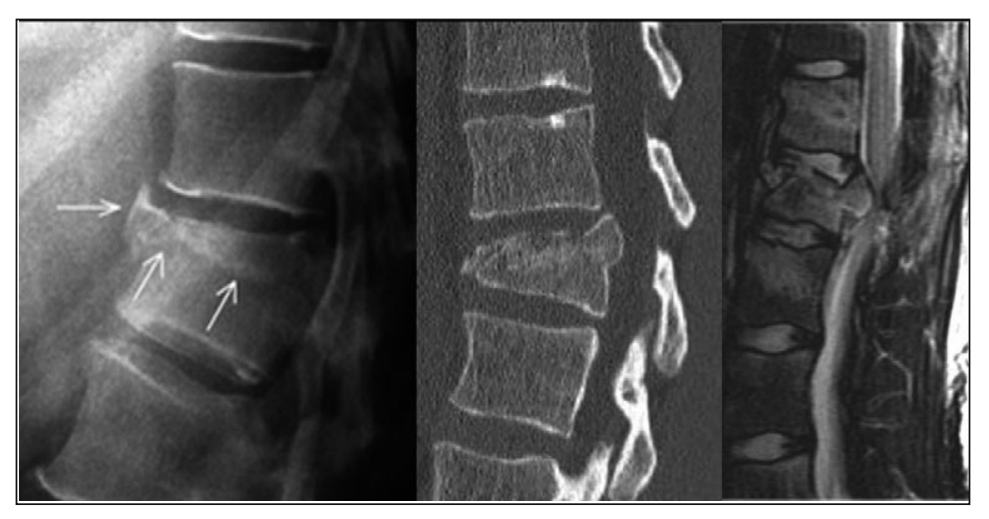
Fig. 3. Frattura instabile mielica ("burst fracture") brali lesionate. Gli stessi appro-

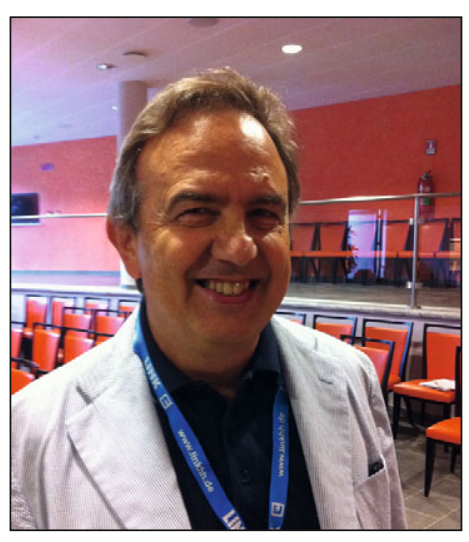

A.Zerbi

tare la sofferenza midollare diffusa e le lesioni focali del midollo, così come le alterazioni delle strutture capsulo-legamentose. Vi è un rapporto stretto tra instabilità di una frattura vertebrale e lesione midollare. Una lesione stabile non è quasi mai una lesione mielica; una lesione instabile può invece essere mielica o

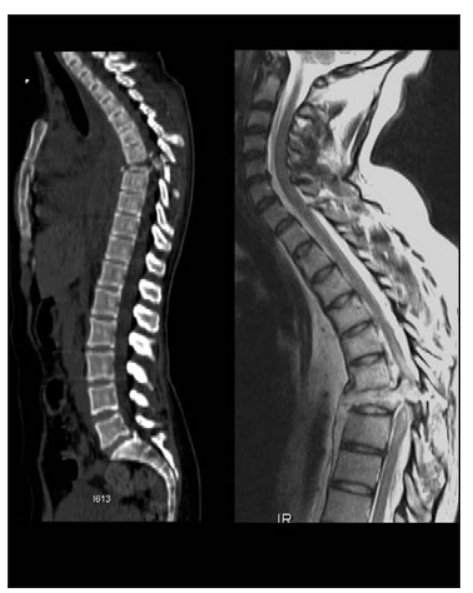

Fig. 4. Frattura da scoppio in politrauma con danno midollare

amielica (Figg. 2,3)

Se una lesione traumatica della colonna vertebrale instabile deve essere trattata chirurgicamente, è necessario sottolineare che una lesione mielica va trattata anch'essa chirurgicamente; è però necessario in questi casi adottare un trattamento chirurgico di emergenza (decompressione $e$ stabilizzazione). Anche in urgenza la RM è fondamentale nella valutazione del danno midollare (Fig. 4).

In conclusione, la RM si pone come esame di scelta nella diagnosi, datazione e classificazione (eventualmente affiancata dall'esame Rx e TC) delle fratture dorso-lombari. In particolare, in emergenza è indispensabile per la valutazione del danno midollare e per la pianificazione della successiva strategia chirurgica.

Letture consigliate

Vialle LR, Vialle E (2005) Thoracic spine fractures. Injury 36[Suppl. 2]:65-72

Parizel PM, van der Zijden T, Gaudino $S$ et al (2010) Trauma of the spine and spinal cord: imaging strategies. Eur Spine J 19[Suppl. 1]:8-17

Looby S, Flanders A (2011) Spine trauma. Radiol Clin North Am 49:129-163

Bagley LJ (2006) Imaging of spinal trauma. Radiol Clin North Am 